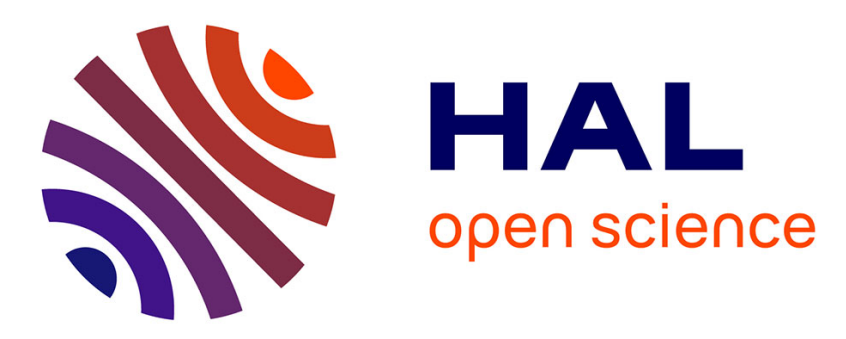

\title{
Les collocations lexicales: une relation essentiellement binaire définie par la relation prédicat-argument
}

\author{
Agnès Tutin
}

\section{To cite this version:}

Agnès Tutin. Les collocations lexicales: une relation essentiellement binaire définie par la relation prédicat-argument. Langages, 2013, Vers une extension du domaine de la phraséologie, 1 (189), pp.4763. 10.3917/lang.189.0047 . hal-01336069

\section{HAL Id: hal-01336069 https://hal.science/hal-01336069}

Submitted on 6 Oct 2018

HAL is a multi-disciplinary open access archive for the deposit and dissemination of scientific research documents, whether they are published or not. The documents may come from teaching and research institutions in France or abroad, or from public or private research centers.
L'archive ouverte pluridisciplinaire HAL, est destinée au dépôt et à la diffusion de documents scientifiques de niveau recherche, publiés ou non, émanant des établissements d'enseignement et de recherche français ou étrangers, des laboratoires publics ou privés. 


\section{Les collocations lexicales : une relation essentiellement binaire définie par la relation prédicat-argument}

\section{INTRODUCTION ${ }^{1}$}

Les collocations lexicales, définies comme des associations lexicales privilégiées et sémantiquement compositionnelles (ex : tristesse infinie, pertes abyssales, jouer un rôle, etc.) constituent désormais une notion essentielle dans les approches contemporaines de la phraséologie. Si l'on reprend cependant les définitions classiques des expressions polylexicales ou des locutions, par exemple celles qui sont proposées par R. Martin (1997) ou B. Lamiroy et al. (2010), ces expressions contribuent toutefois à une extension de ce champ, en tant qu'elles ne sont (a) pas figées pour la plupart sur le plan syntaxique et, d'autre part, (b) tout à fait compositionnelles. Ce qui caractérise ces expressions ressortit plutôt à la fréquence, la spécialisation du sens en contexte et la restriction combinatoire, propriétés qui donnent à ces séquences un caractère préfabriqué et prêt à l'emploi. En outre, ces expressions présentent ceci de remarquable qu'elles sont les plus fréquentes des expressions polylexicales (Moon 1998). De plus, leur facilité de décodage les rend paradoxalement complexes à encoder en langue étrangère (Hausmann 1989 ; Nesselhauf 2005) : le locuteur non natif les comprend, mais ne sait pas les reproduire.

Si la notion est ancienne - on la retrouve déjà chez C. Bally (1909) sous le terme de série phraséolologique - le terme de collocation est lui-même plus

1. Un grand merci à Francis Grossmann et Dominique Legallois pour leurs commentaires avisés sur une première version de cet article. 
récent. Il naît dans le terreau de la didactique du lexique (Palmer 1933) et est rapidement adopté avec une acception plus large dans les modèles fonctionnels anglais, d'abord par J. Firth (1957), et son « meaning by collocation», puis par J. Sinclair (1991) qui le définit comme certains chercheurs du TAL (par exemple, Smadja 1993) avant tout sur une base statistique (cf. aussi Williams 2003 ; Léon 2007 pour une discussion sur le concept dans la tradition anglaise). En Europe continentale, une autre conception, plus formelle et moins fonctionnelle, s'est développée parallèlement. Dans la lignée de C. Bally et de ses « séries phraséologiques », les lexicologues et lexicographes ont proposé des modélisations syntaxiques et syntaxiques du phénomène (cf. Hausmann 1989 ; Grossmann \& Tutin 2003 ; Hausmann \& Blumenthal 2006), axées autour de la distinction de la base, l'élément stable de la collocation, et du collocatif, le satellite du premier élément en quelque sorte. Dans les expressions grièvement blessé et peur bleue, blessé et peur seraient ainsi les bases et grièvement et bleue, les collocatifs, auraient un fonctionnement non autonome, "synsémantique " pour reprendre le terme de F. Hausmann (2004), appelés par l'autre élément de la cooccurrence. C'est cette acception du terme de collocation que nous adoptons dans cet article.

Depuis quelques années, quelques auteurs (par exemple, Bartsch 2004 ; Siepmann 2006) remettent cependant en question la nature exclusivement binaire des collocations et proposent une extension de la notion de collocation lexicale, qui inclurait des suites de mots «n-aires » et se définirait par un fonctionnement pragmatique spécifique. S'il ne fait aucun doute que certaines séquences polylexicales excèdent la structure binaire, nous aimerions montrer dans cet article qu'il existe un sous-ensemble d'expressions lexicalisées dont le fonctionnement est fondamentalement binaire, propriété qui découle de la structure argument-prédicat dans laquelle elles s'insèrent. Il ne s'agit pas pour nous de prétendre que toutes les expressions lexicalisées et compositionnelles peuvent être analysées comme des collocations. Les séquences lexicalisées ne constituent probablement pas un ensemble homogène et les tentatives de définition d'un prototype s'avèrent généralement décevantes ${ }^{2}$. Nous souhaitons ici montrer que, parmi différents types d'expressions lexicalisées, il existe un schéma sémantique et syntaxique particulièrement propice à la lexicalisation et à la spécialisation du sens.

Dans un premier temps, nous revenons sur les propriétés binaires des collocations mises en avant par plusieurs linguistes et le traitement proposé pour ces expressions dans les dictionnaires de collocations. Nous examinons, dans un second temps, quelques cas complexes qui remettent en question la binarité. Nous terminons par une analyse de la structure sémantique des collocations.

2. Les propriétés associant la non-compositionnalité sémantique, le figement syntaxique, l'unité référentielle, la figurativité (cf. Nunberg, Sag \& Wasow 1994 ; Martin 1997) s'avèrent finalement trop limitatives pour circonscrire l'ensemble des expressions perçues comme lexicalisée et les définitions les plus opérationnelles restent souvent les définitions larges de la psycholinguistique (cf. Wray, 2002 : 9, par exemple). 


\section{LES DESCRIPTIONS BINAIRES DE LA COLLOCATION}

\subsection{Deux types de propriétés binaires comme critères définitoires de la collocation}

Les définitions de la notion de collocation dans la tradition continentale la décrivent généralement comme une expression binaire (Hausmann 1989; Heid 1994 ; Mel'čuk 1998). Cette propriété recouvre en réalité deux aspects : d'une part, une binarité catégorielle qui associe deux types de constituants, généralement des unités lexicales ; d'autre part, une binarité fonctionnelle qui indique un fonctionnement dissymétrique, antagonique entre les deux éléments. Ces deux aspects cohabitent dans les définitions de référence de la collocation comme les suivantes :

On appellera collocation la combinaison de deux mots ${ }^{3}$ [...]. Dans la collocation, le statut des deux partenaires combinés n'est pas égal. (Hausmann, 1989 : 1010)

La collocation [...] est la combinaison d'un mot sémiotaxiquement autonome, donc choisi indépendamment de son contexte, que nous appelons base [...], et d'un mot sémiotaxiquement dépendant, choisi en fonction de la base et que nous appelons collocatif. (Hausmann, 2007 : 122-123)

[...] collocations are combinations of exactly two lexemes (of category noun, verb, adjective or adverb), realizing two concepts where the choice of one of them depends on (or: is restricted by) the other. (Heid, $1994: 228)^{4}$

A COLLOCATION AB of language $\mathbf{L}$ is a semantic phraseme of $\mathbf{L}$ such that its signified ' $\mathrm{X}$ ' is constructed out of the signified of one of its two constituent lexemes say, of $\mathbf{A}$ - and a signified ' $C^{\prime}\left[{ }^{\prime} X^{\prime}={ }^{\prime} A \oplus C^{\prime}\right]$ such that the lexeme $\mathbf{B}$ expresses only ' $C^{\prime}$ contingent on A. (Mel'čuk, $1998: 30)^{5}$

La binarité catégorielle met en jeu des mots pleins, qu'il s'agisse de catégories ouvertes (nom, verbe, adjectif ou adverbe) ou de catégories fermées comme des prépositions « pleines » dans certains emplois (par amour, de dépit) ${ }^{6}$. Sont ici

\footnotetext{
3. C'est nous qui mettons en italique.

4. «Les collocations sont des combinaisons d'exactement deux lexèmes (de la catégorie du nom, verbe, adjectif ou adverbe), réalisant deux concepts où le choix de l'un dépend (ou est restreint) par l'autre. » (Heid, $1994: 228)$
}

5. « Une Collocation $A B$ d'une langue $L$ est un phrasème sémantique de $L$ tel que son signifié ' $X$ ' est construit à partir du signifié d'un des deux lexèmes constitutifs - disons $\mathrm{A}$ - et le signifié ' $\mathrm{C}$ ' [' $\mathrm{X}$ ' = 'A $\left.\oplus \mathrm{C}^{\prime}\right]$ tel que le lexème B exprime seulement 'C' en cooccurrence avec A. » (Mel'čuk, 1998 : 30)

6. On observe un certain désaccord sur le traitement des prépositions dans la littérature sur les collocations : certains auteurs comme Hausmann les excluent comme collocatifs, d'autres comme Mel'čuk et Polguère intègrent certaines d'entre elles. On considérera, pour notre part, conformément à certains modèles linguistiques comme la Grammaire Lexicale Fonctionnelle ou la Théorie Sens-Texte, que certaines prépositions peuvent fonctionner comme des mots pleins lorsqu'elles introduisent des modifieurs (et ne sont donc pas régies par une autre catégorie) et comme des mots-outils lorsqu'elles sont régies par un lexème. La même préposition peut remplir les deux fonctions. Par exemple, de dans je ne me souviens de cela sera considéré comme une préposition régie, « incolore » pour reprendre le terme de Spang-Hanssen (1963); en revanche, dans De dépit, elle claqua la porte, où de introduit un complément de phrase de cause, on pourra considérer en revanche de comme une préposition « colorée », sémantiquement pleine. Cf. la discussion § 2.4.2. 
exclus la plupart des mots grammaticaux, dont on considère généralement qu'il s'agit ici de satellites des mots pleins. Les définitions présentées supra peuvent bien entendu être considérées comme trop restrictives si elles n'incluent que des mots « simples ». D'une part, les lexèmes peuvent être eux-mêmes des mots composés ou des phrasèmes figés, pour reprendre la terminologie de I. Mel'čuk comme dans défendre un point de vue ou attendre de pied ferme où point de vue et de pied ferme, peu compositionnels, font office de phrasèmes. D'autre part, certaines expressions non autonomes - et qui, non lexicalisées, ne peuvent prétendre au statut de phrasèmes - comme à pleurer dans triste à pleurer ou comme une tomate dans rouge comme une tomate doivent, selon nous, aussi être intégrées dans la définition de la binarité catégorielle. On pourrait donc reformuler de façon plus précise le critère de binarité catégorielle comme suit : « La collocation met en jeu une unité lexicale simple ou complexe en relation syntaxique avec un constituant linguistique $»$.

La question de la binarité fonctionnelle est plus intéressante. Les définitions supra précisent que les deux éléments n'ont pas le même statut : l'un, souvent appelé la base, conserve son sens habituel. L'autre, le collocatif, est en quelque sorte appelé par le premier. Il est « sémiotaxiquement dépendant, et choisi en fonction de la base » (Hausmann, 2007 : 122-123). Le collocatif peut voir son sens modulé en fonction du contexte : bleu prend un sens intensif en cooccurrence avec peur mais pas avec crainte ou frayeur; faire est le causatif de peur alors que donner est celui de faim ; infiniment s'associe à remercier quand grièvement est préféré avec blesser. Dans ces structures, c'est le deuxième élément qui est le plus inattendu, le plus idiomatique et, si l'expression est bien compositionnelle, il y a souvent une restriction sémantique et/ou lexicale sur ce deuxième élément. Cette dissymétrie n'est pour nous nullement le fruit du hasard. Nous pensons qu'elle est liée à la structure sémantique de la collocation qui s'analyse comme l'association d'un prédicat, le collocatif, associé à un argument plus stable sur le plan lexical et sémantique, la base.

\subsection{Les dictionnaires de collocations : une modélisation du caractère binaire}

Si le caractère binaire des collocations est parfois remis en question par les linguistes (nous examinerons quelques cas problématiques dans la section 2), les lexicographes semblent le considérer suffisamment opérationnel pour l'institutionnaliser dans des dictionnaires destinés au grand public ou à des publics d'apprenants. De nombreux recueils récents de collocations, par exemple le Oxford Collocations Dictionary for Students of English (Crowther, Dignen \& Lea 2002) ou le Dictionnaire des combinaisons de mots (Le Fur 2007), mais aussi des ouvrages anciens comme Les mots et les idées. Dictionnaire des termes cadrant avec les idées (Lacroix 1931), entérinent, conformément aux vœux de F. Hausmann (1989) cette 
vision binaire et dissymétrique de la collocation ${ }^{7}$. L'expression y est toujours codée dans tous ces ouvrages sous l'entrée de la base (Tutin 2010) ${ }^{8}$. Ainsi, par exemple, comme on le voit Figure 1, dans le dictionnaire de U. Lacroix, c'est sous l'entrée de «chance » que l'on trouver tenter sa chance, chance inespérée, incroyable, etc.

Chance. Tenter, courir la chance. Posséder, augmenter, diminuer, réduire, supputer, calculer ses chances. Les chances augmentent, diminuent. La chance toume.QUAL. : grande, maigre, mince, inespérée, inouïe, incroyable, immense, énorme, nombreuse.

Figure 1 : Un exemple d'entrée dans un dictionnaire de collocations : Les mots et les idées (Lacroix 1931)

Dans le domaine de la lexicographie expérimentale, le modèle des Fonctions Lexicales de la Lexicologie Explicative et Combinatoire (Mel'čuk, Clas \& Polguère 1995 ; Mel'čuk 2003), qui est sans conteste la modélisation la plus ambitieuse et la plus aboutie dans la tradition continentale (cf. aussi Wanner 1996), propose également une analyse dichotomique des collocations ${ }^{9}$. Elles y sont décrites à l'aide d'opérateurs abstraits, les Fonctions Lexicales, rendant compte du comportement syntaxique et sémantique de l'association lexicale : Magn par exemple symbolise la relation d'intensité associant à une lexie (la base) son modifieur (le collocatif) - p. ex. Magn $($ peur $)=$ bleue; Magn $($ travailler $)=d^{\prime}$ arrache-pied,

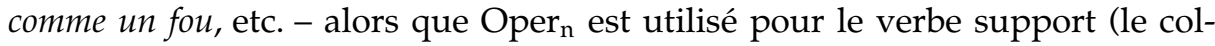
locatif) introduisant le nom prédicatif (la base), p. ex. Oper ${ }_{1}($ échec) $=$ essuyer ; Oper $_{1}$ (haine) $=$ concevoir $($ cf. discussion § 3 ).

En bref, le codage binaire et dissymétrique de ces expressions (le codage de l'expression sous la base) a été d'une certaine façon institutionnalisé par l'usage lexicographique, et si ces ouvrages ont rencontré leur public (certains d'entre eux comme le Oxford Collocations Dictionary for Students of English (Crowther, Dignen \& Lea 2002) ou le Dictionnaire des combinaisons de mots (Le Fur 2007) sont publiés dans des maisons d'édition reconnues (Oxford University Press ou Le Robert)), on peut faire raisonnablement l'hypothèse qu'il n'est pas improbable que cette propriété soit appréhendée intuitivement. Du côté des linguistes, en revanche, le caractère binaire de ces expressions est parfois remis en question.

7. Le plus ancien dictionnaire monolingue du français selon Hausmann \& Blumenthal (2006) est d'ailleurs un dictionnaire de collocations organisé selon ce principe. Les épithètes de M. de La Porte (de 1571) répertoriait 80000 collocations adjectif-nom réparties dans les articles de 4000 substantifs.

8. Le dictionnaire de Grobelak (1990), Dictionnaire collocationnel du français général, propose toutefois un double codage, à la fois sous la base et sous le collocatif.

9. Les phrasèmes figés font, en revanche, l'objet d'une entrée à part entière. 


\section{QUELQUES CAS INTÉRESSANTS}

Certains auteurs comme S. Bartsch (2004) ou D. Siepmann (2006) contestent le caractère binaire des collocations sur deux plans : $d$ 'une part, parce que certaines expressions lexicalisées et compositionnelles dépassent l'association binaire (binarité catégorielle) ; d'autre part, parce qu'il n'apparaît pas possible de mettre en évidence un élément dominant dans l'association (binarité fonctionnelle). En d'autres termes, dans certains cas, la distinction entre base et collocatif ne serait pas toujours possible.

En ce qui concerne la binarité catégorielle, de nombreux cas considérés comme épineux par ces auteurs peuvent être ramenés après analyse à des structures binaires (cf. aussi Tutin 2008). Si l'on observe en effet bien des structures n-aires (ici ternaires) comme dans avoir un geste déplacé (Siepmann 2006), to pay close attention ou freshly baked bread ${ }^{10}$, il s'agit généralement du résultat de constructions binaires qui se combinent dans plusieurs opérations de la même façon qu'en morphologie constructionnelle, les opérations d'affixation peuvent se succéder ${ }^{11}$. Ces combinaisons de collocations, assez courantes dans la langue, sont décrites par F. Hausmann (2007) à l'aide du terme de « chaîne collocationnelle", alors que D. Spohr (2005) emploie celui de "collocational cluster ». Examinons de plus près quelques exemples où ces opérations sont à l'œuvre.

\subsection{Deux modes de combinaisons des collocations}

\subsubsection{La superposition de collocations}

Une expression comme avoir une peur bleue se décompose naturellement en deux collocations autonomes : avoir peur et peur bleue. Les deux expressions, indépendantes, sont ici superposées du fait de leur base commune, le mot peur. Il est vrai que peur bleue dans les corpus apparaît préférentiellement avec avoir (19 occurrences sur 25 dans un sous-corpus de langue contemporaine de Frantext que nous appelons ici Frantext $1{ }^{12}$ ), mais la collocation n'est pas impossible dans un autre contexte, comme l'attestent les exemples suivants. L'existence de ces collocations indépendantes dans les corpus prouve que l'on est bien en présence d'une superposition de collocations :

(1) Dans le couloir, je me suis fait une peur bleue, je me suis vu digéré par un boa constrictor. (Djian, 37.2 le matin, 1985)

10. C'est nous qui soulignons.

11. L'association collocative présente d'ailleurs plusieurs points communs avec l'affixation : a) elles sont toutes les deux binaires ; b) elles associent un sens plus pauvre et générique à un sens plus riche (l'affixe à la base comme le collocatif est associé à la base lexicale); c) elles fonctionnent essentiellement sur une structure sémantique prédicat-argument (l'affixe est le prédicat de la base, le collocatif est le prédicat de la base).

12. Frantext1 : Frantext, prose dans la base catégorisée de 1900 à 2007, soit 70219581 mots. 
Il se tourna vers moi et me regarda avec une peur bleue, à cause des émotions que ça allait lui causer. (Ajar, La vie devant soi, 1975)

Plusieurs cas souvent considérés comme complexes peuvent être analysés de la même façon. D. Siepmann (2006 : 102) cite ainsi le cas de avoir un geste déplacé qui, selon lui, ne peut pas être décomposé, car *avoir un geste n'est pas attesté. En effet, pour ces collocations, il apparaît difficile de supprimer un des éléments :

(3) a. Il a eu un geste déplacé.

b. ?*Il a eu un geste.

Cependant, comme pour peur bleue, geste déplacé peut apparaître en dehors du contexte d'avoir. Dans Frantext1, on relève ainsi trois occurrences de cette expression, comme la suivante, dont aucune n'apparaît en cooccurrence avec avoir :

(4) Une des filles, plutôt mignonne, emmitouflée dans une veste matelassée, était assise sur les genoux d'un consommateur qui ne se permettait aucun geste déplacé. (Hanska, Les amants foudroyés, 1984)

En outre, le verbe support et le nom peuvent apparaître en dehors du contexte de déplacé, comme dans l'exemple suivant :

(5) Elle aurait eu ce geste pour éloigner d'elle le journal, pour regarder à bout de bras les dessins que griffonnent les enfants les jours d'orage où les mouches s'affolent... (Nourissier, Le maître de maison, 1968)

Certaines contraintes syntaxiques s'observent bien avec avoir, puisque le verbe est ici toujours accompagné d'un modifieur, que celui-ci se réalise par un adjectif, une relative ou un syntagme prépositionnel (sans qu'il y ait une contrainte spécifique sur la lexicalisation du modifieur). Ces contraintes syntaxiques et sémantiques doivent être associées au verbe support avoir et ne sont donc pas caractéristiques de l'association sémantique en jeu. Autrement dit, avoir un geste déplacé peut donc bien être interprété comme la superposition de la collocation avoir un geste + Modifieur et geste déplacé. Les contraintes syntaxiques associées aux collocations peuvent, en outre, expliquer pourquoi certaines collocations ne peuvent pas être fusionnées. Par exemple, contrairement à avoir peur, prendre peur ne peut pas être fusionnée avec peur bleue : *prendre une peur bleue. La différence est liée aux structures syntaxiques des collocations. Alors que avoir peur admet une construction syntaxique comme V DET N + Modifieur, tel n'est pas le cas de prendre peur, qui n'admet aucune modification, probablement parce que la valeur inchoative $\mathrm{du}$ verbe bloque en quelque sorte la modification. La plupart des collocations apparemment tripartites (jouer un rôle clé, to pay close attention, etc.) sont analysables en termes de superposition de collocations.

\subsubsection{L'enchâssement de collocations}

Le cas des superpositions de collocations doit être distingué des cas d'enchâssement de collocations (ou collocations " récursives »), lorsque le collocatif ou la base peuvent eux-mêmes être des collocations. Dans tomber dans $l^{\prime}$ oubli, le collocatif tomber (verbe support à valeur inchoative) porte, en effet, sur 
la collocation dans l'oubli qui comporte elle-même le collocatif dans qui exprime en quelque sorte l'état ${ }^{13}$. La collocation dans l'oubli est tout à fait productive dans d'autres contextes, par exemple comme attribut du verbe être ou comme épithète, comme dans l'exemple (6) extrait de Frantext1 :

(6) C'était un lieu damné qu'on laissait dériver dans l'oubli. L'odeur n'avait pas changé. Une odeur de chien mort corsée par le moisi. (Quéfellec, Les noces barbares, 1985)

Le collocatif tomber s'applique ici à la collocation dans l'oubli tout entière (de la même façon que son synonyme moins productif s'enfoncer). La même analyse s'applique à tomber en amour (Fr. québécois), même si le verbe tomber paraît plus productif comme collocatif d'adjectif exprimant le changement d'état subit (tomber malade, tomber amoureux, tomber enceinte).

Parallèlement, une collocation pourra avoir comme collocatif une collocation. C'est à notre avis de cette façon que doit être analysée la séquence freshly baked bread considérée par S. Bartsch (2004:67) comme une séquence unique. $\mathrm{S}$. Bartsch considère ainsi que tous les éléments de cette expression sont indissociables. Cependant, on remarque que la séquence freshly baked est très productive en anglais. Une recherche rapide sur le corpus COCA (The Corpus Of Contemporary American English de 450 millions de mots ${ }^{14}$ ) nous fournit 160 occurrences de cette association, et confirme la productivité de freshly baked bread(s) (47 occurrences), mais on relève aussi freshly baked cookies (9 occ.), freshly baked rolls (4 occ.), freshly baked pie(s) (4 occ.), etc. ; freshly baked peut ainsi lui-même être considéré comme une collocation (l'adverbe freshly s'associe préférentiellement à baked), la collocation elle-même étant utilisée comme collocatif de bread.

De nombreuses collocations se combinent donc par superposition ou enchâssement (cf. aussi Alonso Ramos \& Wanner 2007 pour d'autres types de combinaisons) : le résultat de ces opérations produit des expressions n-aires qui peuvent elles-mêmes être très productives sous cette forme, comme avoir une peur bleue. Il faut donc dissocier selon nous l'opération de construction de ces expressions (qui s'articule autour de la binarité) du résultat de cette construction ${ }^{15}$. Pour des raisons pratiques cependant, qu'il s'agisse d'applications lexicographiques ou de traitement automatique des langues, il peut s'avérer utile ou même indispensable de consigner certaines de ces constructions n-aires qui seraient productives (p. ex. jouer un rôle clé, avoir une faim de loup, etc.). Cela ne remet nullement en question pour nous dans la plupart des cas le caractère binaire de ces associations.

13. Sur les constructions verbales avec la préposition dans, voir Vaguer 2006.

14. http://corpus.byu.edu/coca/

15. Comme en morphologie constructionnelle. 


\subsection{Questions sur la dissymétrie des collocations}

Certaines expressions s'apparentent à des collocations (ce sont des associations privilégiées, binaires et compositionnelles), mais il apparaît difficile de distinguer l'élément qui fait office de base de l'élément qui serait le collocatif (Bartsch 2004 ; Siepmann 2006 ; Hausmann 2007). Il est intéressant d'observer que ces critiques se concentrent principalement sur deux structures : $\mathrm{N}$ prep $\mathrm{N}$ (p. ex. crise d'angoisse, meute de loups) et $\mathrm{V}$ prep $\mathrm{N}$ (p. ex. rougir de honte, cf. Hausmann 2007) que nous examinerons tour à tour.

\subsubsection{Les structures à nom quantitatif ( $\mathrm{N}_{\text {Quant }}$ de $\left.\mathrm{N}\right)$ : "une meute de loup"}

Dans les expressions comme meute de chiens (ou a pack of dogs ${ }^{16}$ pour l'anglais) ou une crise d'angoisse, il est bien connu que deux interprétations sémantiques (et syntaxiques) sont possibles (cf. Milner 1978 ; Flaux \& van de Velde 2000). Si l'on met l'accent sur l'interprétation quantitative («Mult» dans le langage des Fonctions Lexicales de Melč'uk pour meute ou pack, ou « Sing » pour crise), alors la base est chiens ou angoisse, le collocatif étant l'élément quantitatif. Par syllepse, le nombre et le genre du SN sont déterminés par l'élément quantitatif (une meute de loups ont attaqué ${ }^{17}$ ). En revanche, si l'on choisit l'interprétation non quantitative, alors la base est meute ou crise et chien ou angoisse sont des compléments de noms régis. L'accord en genre et en nombre se fait avec le nom recteur et son déterminant (une meute de loups a attaqué). L'hésitation entre les deux noms est finalement liée à une ambiguiité présente dans la langue. Si l'on analyse l'expression comme une collocation, où certains éléments sont désémantisés, alors l'interprétation du premier nom comme un élément quantitatif est plus naturelle.

\subsubsection{Les structures $\mathbf{V}$ de $\mathbf{N}$ : 'rougir de honte" ${ }^{18}$}

Certaines structures $\mathrm{V}$ de $\mathrm{N}$ peuvent elles aussi prêter à deux interprétations syntaxiques et sémantiques : trembler de peur, rougir de honte... (cf. Hausmann, 2007 : 110). Comme le montre D. Leeman (1991), ces structures qui introduisent souvent des compléments de cause peuvent correspondre à plusieurs emplois. À un extrême, la structure de N peut être assez autonome et autoriser le déplacement, comme dans les exemples où elle apparaît en tête de phrase :

(7) De honte, elle irait se jeter dans la pièce d'eau. (Rheims, Les greniers de Sienne, 1987)

16. Examiné par Bartsch (2004 : 36).

17. Cet emploi est bien décrit dans le Bon Usage (Grevisse, $11^{\mathrm{e}}$ éd., 1980 : 949) : «L'accord a lieu avec le complément (accord avec le sens : syllepse du nombre) si l'on a en vue la pluralité des êtres dont il s'agit, considérés individuellement ». Les exemples sont multitude, foule, poignée, troupe, bande, peuple, troupeau, couple, troupeau.

18. La même analyse peut probablement se généraliser à d'autres structures V Prép N. 
(8) De colère, il a cassé le pare-brise de la voiture qu'il réparait. (Thérame, Bastienne, 1985)

Dans ce cas, le complément de cause porte sur le verbe et le nom peut être considéré comme le collocatif. À l'autre extrémité, le verbe est très désémantisé (métaphore, hyperbole) et deviendrait un « modifieur du nom » selon les termes de D. Leeman. Dans ce cas, le complément de cause n'est plus déplaçable (*de colère, il bout; *de rage, il blanchit). Ici, le collocatif est bien le verbe et le nom la base. S'il est si difficile de déterminer dans certaines associations comme rougir de honte si le verbe est le collocatif ou la base, c'est que l'ambiguité est ici présente. Les deux structures sont, en effet, possibles, selon que l'on souhaite mettre l'accent sur la manifestation physique (la honte est véritablement la cause de la rougeur) ou sur l'affect même (dans ce cas, le verbe rougir est une des manifestations de la honte et son sens peut être atténué). Dans certaines expressions comme mourir de peur, l'ambiguïté est encore plus nette, bien que l'interprétation hyperbolique soit la plus naturelle et la plus productive.

En bref, les cas de figure examinés ne remettent pas véritablement en question la structure dissymétrique des collocations. Si certaines hésitations sont possibles dans certaines structures, c'est parce que celles-ci présentent une ambiguïté sémantique et syntaxique : l'un des éléments (le premier nom dans la structure $\mathrm{N}$ de $\mathrm{N}$, ou le verbe dans la structure $\mathrm{V} d e \mathrm{~N}$ ) peut être analysé comme une sorte de modifieur, souvent désémantisé, ou bien véritablement comme un mot plein recteur de la construction. Dans la première interprétation, on a généralement bien affaire à une collocation et le premier élément peut généralement être analysé comme un collocatif.

Au terme de l'examen de cas souvent considérés comme délicats, force est donc de constater que la binarité et la dissymétrie semblent bien être des propriétés prototypiques pour la fabrication d'un modèle d'expressions. Comment expliquer toutefois la productivité de ce moule binaire ? Favorise-t-il la lexicalisation et pour quelles raisons? Nous essayons de proposer des éléments de réponse dans la section suivante.

\section{LA STRUCTURE PRÉDICAT-ARGUMENT COMME MOULE PHRASÉOLOGIQUE DE LA COLLOCATION}

\subsection{L'analyse de la collocation comme structure prédicat-argument}

Si la binarité semble donc bien caractéristique d'un ensemble productif d'expressions lexicalisées, cette structure n'est pas arbitraire, mais étroitement liée à la structure sémantique prédicat-argument sous-jacente. Dans la collocation, le collocatif, l'élément souvent inattendu dans sa réalisation lexicale, fait office de prédicat : il exprime une propriété, un état, une action au sujet de l'élément qui l'accompagne. Ainsi, bleue indique l'intensité dans peur bleue, comme pour mourir dans mourir de peur. Le verbe faire dans faire peur correspond à la notion 
de cause, essuyer associé au nom échec permet d'actualiser le nom prédicatif. Le second élément de la collocation, plus stable sur le plan lexical, s'interprète, lui, de façon " autosémantique ", sans prendre en compte l'environnement lexical.

Les collocations, organisées autour de la structure argument-prédicat, sont donc compositionnelles dans la mesure où il est possible d'associer à chaque élément de l'expression un contenu sémantique et de calculer le sens de l'ensemble à partir des composants. Ce critère de compositionnalité - il est vrai souvent difficile à manier (cf. Nunberg, Sag \& Wasow 1994 ; Svensson 2004) permet de distinguer les collocations d'un ensemble d'expressions plus figées pour lesquelles la compositionnalité n'est pas accessible en synchronie : pied noir est clairement non compositionnel contrairement à pied grec dans la mesure où le collocatif grec indique une propriété du référent dénoté par pied.

Le critère de prédication nous paraît en outre pertinent pour distinguer les collocations de certaines compositions nominales, la discrimination entre ces deux types d'expressions étant parfois particulièrement délicate, surtout lorsque les composés nominaux apparaissent partiellement compositionnels. En effet, si des expressions comme vin charpenté/jeune ou vin blanc/rouge, sont toutes binaires et composées de la même façon d'un nom et d'un adjectif, elles n'ont pas du tout le même fonctionnement sémantique (et référentiel), au-delà de leur similitude formelle (cf. aussi la discussion sur vin blanc dans Gross, 1996 : 50). Le nom vin s'interprète dans tous les cas de façon littérale, contrairement aux adjectifs dont le sens est étroitement dépendant de celui du nom. Dans la première série d'expressions (vin charpenté/jeune), l'adjectif prédique une qualité du référent et l'expression est parfaitement compositionnelle. Il se détache assez facilement du nom et peut s'appliquer à des sous-catégories du nom, par exemple bordeaux pour vin.

(9) Ce vin est (charpenté + jeune)

(10) Ce bordeaux est (charpenté + jeune)

En revanche, blanc et rouge associés au nom vin n'indiquent pas une qualité, mais un type. Ce ne sont pas des prédicats ici (même s'ils ont pu l'être dans un état de langue antérieur), mais des éléments qui servent à affiner la catégorisation évoquée par le nom. L'emploi comme attribut ou avec des exemplaires apparaît incongru. On préfère ici substantiver l'adjectif (un rouge, un blanc) ce qui montre bien la fonction catégorisante, plutôt que prédicative, de l'adjectif.

(11) a. ?Ce vin est (rouge + blanc)

b. Ce vin est (un rouge + un blanc)

(12) a. ?Ce bordeaux est (rouge + blanc)

b. Ce bordeaux est (un rouge + un blanc)

Au plan référentiel, vin rouge et vin blanc renvoient à des types de vin, propriété qui les rapproche des noms composés. L'adjectif sert ici à créer une dénomination, un terme. Bien entendu, un glissement est toujours possible d'une qualité vers une catégorie référentielle, lorsque les conditions extralinguistiques sont réunies, par exemple dans un sous-langage spécifique. Le terme vin moelleux par exemple 
est relativement équivoque dans la mesure où il peut à la fois désigner une qualité (ce vin est moelleux), mais aussi une catégorie (Les vins moelleux du sud ouest sont les plus appréciés). En bref, le critère de prédication contribue à la compositionnalité des collocations et fournit un indice supplémentaire pour isoler ces expressions des compositions, même si la frontière est parfois poreuse.

Une observation empirique vient étayer notre analyse des collocations comme structures prédicat-argument. Dans les dictionnaires de collocations, on observe que la majorité des collocations est organisée autour d'une base nominale, que le collocatif soit un adjectif (célibataire endurci), un verbe (jouer un rôle) ou un autre nom (un nuage de lait). Les répertoires " grand public » de collocations du français (Dictionnaire des combinaisons de mots ; Dictionnaire des cooccurrences ; Dictionnaire des combinaisons de mots) n'intègrent d'ailleurs que ce type de base. Les dictionnaires de l'anglais, en particulier le BBI Dictionary of Selected Word Combinations ou le Oxford Collocations Dictionary for Student of English, incluent également des bases adjectivales (heavily pregnant, deliberately provocative) ou verbales (to improve dramatically, to care deeply...), mais la catégorie nominale y reste très majoritaire. Cette surreprésentation des noms n'a rien d'étonnant et est liée à la structure sémantique des collocations. Les prédicats sont très largement représentés dans la langue française par des adjectifs et des verbes, au fonctionnement synsémantique (cf. Hausmann 2007), qui portent sur la catégorie nominale : leur sens comporte un noyau sémantique, mais également un élément externe, l'argument sur lequel il porte. La plupart des prédicats portant sur des éléments nominaux, il est donc naturel que ces derniers soient surreprésentés dans ces ouvrages où les entrées sont les bases et non les collocatifs. Cela n'empêche nullement les verbes et les adjectifs d'être eux-mêmes les arguments de prédicats, très souvent dans une relation d'intensité (immensément riche, travailler comme un fou), mais ce cas de figure est plus limité.

\subsection{Des schémas sémantiques productifs}

Les collocations s'inscrivent dans relations sémantiques récurrentes. C. Bally dans son Traité de stylistique française (et dans le volume d'exercices qui l'accompagne) illustrait principalement les "séries phraséologiques » par deux types d'expressions, les " séries d'intensité » (p. ex. chaleur suffocante, diamétralement opposé, refuser catégoriquement) et les " périphrases verbales », des "locutions qui périphrasent des verbes » (Bally, 1909: 72) comme remporter une victoire (=vaincre), prendre une décision (= décider). Les périphrases verbales de C. Bally préfigurent pour la plupart la notion contemporaines de « verbe support » telle que définie par M. Gross et ses collègues (cf. Vivès 1993), i.e. des verbes qui actualisent des noms prédicatifs et sont désémantisés en cooccurrence avec ces noms (faire une promenade, donner un cours, avoir faim...). 
Ces deux types sémantiques de collocations sont très souvent présentés pour illustrer les collocations prototypiques. Dans le cadre de la Lexicologie Explicative et Combinatoire, les Fonctions Lexicales syntagmatiques les plus emblématiques (parmi la trentaine existante) sont sans conteste la fonction Magn qui code l'intensité (Magn(travailler) = comme un fou, d'arrache-pied...); Oper $_{n}$ qui correspond au verbe support $\left(\mathrm{Oper}_{1}(\right.$ demande $)$ : adresser, faire $; \mathrm{Oper}_{2}$ (visite $)=$ recevoir) ; CausFunc $0 / 1$ qui renvoie au verbe causatif $\left(\right.$ CausFunc $_{0 / 1}($ faim $)=$ donner ; CausFunc $_{0 / 1}(\text { peur })^{19}=$ faire), etc. Si ces fonctions lexicales sont si souvent citées, ce n'est pas selon nous seulement pour leurs vertus pédagogiques - ce sont probablement les plus simples à appréhender ${ }^{20}$ - mais aussi parce qu' elles correspondent à des fonctions sémantiques particulièrement productives pour les collocations. Une étude de corpus menée sur le lexique des émotions confirme cette intuition (Augustyn \& Tutin 2009). L'annotation des collocations dans un corpus littéraire de 670000 mots à l'aide de fonctions lexicales permet de mettre au jour des résultats intéressants : d'une part, une écrasante majorité de collocations (94\%) peut être codée par une fonction lexicale standard, ce qui montre la validité empirique de ce modèle, en tout cas pour le corpus et le lexique considérés ${ }^{21}$; d'autre part, on observe que trois fonctions lexicales (Magn, Oper, CausFunc) représentent à elles seules plus de la moitié des occurrences ${ }^{22}$. Ces relations sémantiques paraissent universelles : elles appartiennent d'ailleurs toutes trois à l'ensemble de primitives mises en évidence par A. Wierzbicka (1996) dans sa métalangue sémantique naturelle : 'très' (qui correspond à intensité ou la fonction Magn), 'cause' (qui se réalise dans le verbe causatif ou CausFunc), 'faire' (qui peut correspondre au verbe support ou Oper). Cela nous conduit à penser que, si les collocations s'appuient bien sur une relation prédicat-argument, certains prédicats sémantiques sont plus susceptibles de se réaliser comme des collocatifs, probablement du fait de leur très grand vague sémantique qui conduit à préférer des lexicalisations idiosyncrasiques. Pour réaliser des sens primitifs et universels, la langue semble ainsi contourner l'usage de lexèmes trop génériques comme très, beaucoup, causer, faire et préférer des emplois plus colorés, ressortissant à l'expérience comme les structures comparatives ou des qualificatifs figurés : rouge comme une tomate, mourir de peur, bête à pleurer, pertes abyssales.

\subsection{Le schéma prédicat-argument et la spécialisation du sens}

Si les lexicalisations dans les collocations présentent une part d'idiosyncrasie, elles répondent à des schémas syntaxiques et sémantiques productifs. On peut ainsi proposer une gradation du caractère idiosyncrasique en se basant sur

19. CausFunc ${ }_{0 / 1}$ : 'causer que le sentiment se manifeste (chez qqn)'

20. Le décodage de certaines fonctions composées ou complexes demande une véritable expertise.

21. Le champ sémantique considéré est celui des affects où les relations d'intensité et de cause sont particulièrement présentes (et où, d'une manière générale, la combinatoire est très riche). Une étude de plus grande ampleur sur l'annotation des collocations doit être entreprise pour confirmer ces résultats.

22. Oper $_{1}(25 \%)$, Magn (20\%), CausFunc $0 / 1$ (12\%). 
les propriétés du collocatif (le sens de la base restant généralement standard) (cf. Tutin \& Grossmann 2002). Les collocations « opaques » comme peur bleue comporteraient des collocatifs peu prédictibles (et difficilement décodables) même si une forme de compositionnalité est à l'œuvre (bleue exprime bien ici l'intensité). Les collocations " transparentes » sont décodables, mais a priori peu prédictibles, en tout cas dans leur réalisation lexicale. Ce serait par exemple le cas de collocations répondant à des schémas constructionnels (ou des « matrices lexicales » pour reprendre le terme de J.-C. Anscombre) qui se spécialisent dans des fonctions sémantiques spécifiques : les constructions à comparatif (Adj comme $\mathrm{N}$ : beau comme un cour, $\mathrm{V}$ comme $\mathrm{N}$ : rire comme un bossu, $\mathrm{N}$ de $\mathrm{N}$ : appétit d'ogre ; Adj à Vinf : triste à mourir, $\mathrm{V}$ à Vinf : rire à pleurer) génèrent un sens intensif, à tel point que des associations inédites utilisant ces moules peuvent conserver cette fonction intensive (Mejri 1994). Les collocations "régulières " enfin sont décodables et paraissent en partie prédictibles dans la mesure où il apparaît possible de justifier la cooccurrence par des affinités sémantiques entre les éléments, même si l'analyse n'est pas toujours immédiate. Par exemple, affreux, comme de nombreux adjectifs négatifs, se désémantise et tend à devenir intensif en cooccurrence avec un nom de même polarité, par dans désespoir affreux (Grossmann \& Tutin 2007).

D'une manière générale, quel que soit le degré d'arbitraire du collocatif, on observe donc dans les cas de collocations une spécialisation du sens en contexte : le sens du collocatif se module en fonction de l'élément sur lequel il porte. Cela est tout particulièrement perceptible pour les adjectifs dont il a été souvent noté qu'ils avaient un fonctionnement si spécifique qu'il permet à certains comme R. Lahav (1989) de remettre en question la thèse de la compositionnalité sémantique $^{23}$. R. Lahav souligne ainsi que la plupart des adjectifs, même les adjectifs de couleur, ne peuvent se définir en dehors de l'objet sur lequel ils portent. Ces catégories dépendent sémantiquement du nom, et de ce point de vue, on pourrait presque considérer que tous les adjectifs ont un fonctionnement collocatif, analyse qui nous paraît toutefois un peu radicale ${ }^{24}$. Ce principe semble d'une certaine façon poussé à l'extrême dans le cas des collocations : ici, les adjectifs se spécialisent très fortement en fonction du nom ou du sous-ensemble de noms sur lequel il porte. Nous pensons, en outre, que ce fonctionnement lexico-sémantique est particulièrement à l'œuvre pour des prédicats qui expriment des notions universelles et primitives, comme l'intensité ou la cause. Enfin, il nous semble que les verbes, en tant que prédicats, répondent eux aussi, mais probablement à un degré moindre, aux mêmes caractéristiques que les adjectifs.

23. Auquel nous ne souscrivons pas. Si la lexicalisation apparaît idiosyncrasique, il y a bien une forme de compositionnalité.

24. Un adjectif comme intelligent conservera, nous semble-t-il, à peu près le même noyau sémantique s'il porte sur un nom de type humain. 


\section{POUR CONCLURE}

$\mathrm{Au}$ terme de cet examen, on peut donc constater qu'il existe bien un sousensemble productif d'expressions lexicalisées binaires, organisées autour d'une structure prédicat-argument. Dans ces expressions, ce sont bien les prédicats, dans la majorité des cas des verbes et des adjectifs, qui, non autonomes, spécialisent leur sens en fonction de l'argument, et tout particulièrement quand la notion sémantique à exprimer renvoie à un sens primitif comme l'intensité ou la cause. Ces propriétés justifient bien selon nous le traitement dissymétrique proposé dans les dictionnaires de collocations et le traitement différencié entre collocations et phrasèmes figés de la Lexicologie Explicative et Combinatoire.

Bien entendu, les propriétés mises en évidence ne permettent pas de caractériser l'ensemble des expressions polylexicales - dont nous pensons d'ailleurs qu'il est illusoire de proposer un prototype tant les phénomènes sont divers - mais $\mathrm{d}$ 'isoler les collocations des expressions véritablement figées comme les noms composés. Si la structure prédicat-argument est bien spécifique de la collocation, la binarité en revanche est à l'œuvre dans d'autres types de séquences lexicalisées. Elle s'observe de façon récurrente dans les proverbes et dictons (qui vivra verra ${ }^{25}$, aide-toi, le ciel t'aidera...) et cela de façon universelle (Milner 1969). Elle est également présente dans les « binomials » ou expressions coordonnées comme mort ou vif, aller et retour, sain et sauf, ici et là (Lambrecht 1984) et est bien entendu centrale dans la composition nominale (Benveniste 1974). La binarité semble donc bien constituer un principe universel dans la construction de séquences lexicalisées, qui, au-delà des idiosyncrasies de surface, répondent souvent à des principes d'organisation réguliers.

\section{Références}

Alonso Ramos M. \& WANNer L. (2007), “Collocation Chains: How to Deal with Them?”, Wiener Slawistischer Almanach, Sonderband 69, 11-20.

AugustYN M. \& TUTIN A. (2009), "Constitution d'un corpus annoté autour du lexique des émotions : collocations et fonctions lexicales ", in D. Beck et al. (eds), Proceedings of the Fourth International Conference on Meaning-Text Theory (MTT'09), Montréal: OLST, 25-34.

Bally C. (1909), Traité de stylistique française, Paris : Klincksieck.

BARTSCH S. (2004), Structural and functional properties of collocations in English: A corpus study of lexical and pragmatic constraints on lexical co-occurrence, Tübingen: Narr.

BenVenISTE E. (1974), "Fondements syntaxiques de la composition nominale ", Problèmes de linguistique générale, Paris : Gallimard, 145-162.

CROWTHER J., Dignen S. \& LEA D. (eds) (2002), Oxford Collocations Dictionary for Students of English, Oxford: Oxford University Press.

FIRTH J. R. (1957), Papers in Linguistics 1934-1951, London: Oxford University Press.

25. Merci à Claude Buridant pour cette observation lors d'un séminaire LiLPa à Strasbourg. 
FLAuX N. \& VAN DE VeLDE D. (2000), Les noms en français, esquisse de classement, Paris: Ophrys.

Grobelak L. (1990), Dictionnaire collocationnel du français général, Varsovie : Państwowe Wydawnictwo Naukowe.

GRoss G. (1996), Les expressions figées en français : noms composés et autres locutions, Paris : Ophrys.

Grossmann F. \& TUtin A. (2007), "Motivation of Lexical Associations in Collocations: the Case of Intensifiers denoting 'Joy"', in L. Wanner (ed.), Selected Lexical and Grammatical Issues in the Meaning-text Theory: in Honour of Igor Mel'čuk, Amsterdam/Philadelphia: John Benjamins, 139-165.

Grossmann F. \& TUtin A. (éds) (2003), Les collocations : analyse et traitement, Travaux et recherches en linguistique appliquée, Amsterdam : de Werelt.

HAUSMANN F. J. (1989), "Le dictionnaire de collocations ", in F. J. Hausmann et al. (eds), Wörterbücher/Dictionaries. Ein internationales Handbuch zur Lexikographie/An International Encyclopedia of Lexicography, Berlin/New-York: de Gruyter, 1010-1019.

HaUSmann F. J. (2004), “Was sind eigentlich Kollokationen?”, in K. Steyer (ed.) Wortverbindungen - mehr oder weniger fest, Institut für Deutsche Sprache Jahrbuch 2003, Berlin/New York: De Gruyter: 309-334.

Hausmann F. J. (2007), "Lexicographie française et phraséologie ", in E. Haag (éd.), Collocations, phraséologie, lexicographie. Études 1977-2007, Aachen : Shaker Verlag, 121-153.

Hausmann F. J. \& Blumenthal P. (2006), "Présentation : collocations, corpus, dictionnaires ", Langue française 150, 3-13.

HEID U. (1994), “On Ways Words Work Together - Topics in Lexical Combinatorics”, EURALEX'94 Proceedings, Amsterdam: Vrije Universiteit Amsterdam, 226-257.

LACROIX U. (1931), Les mots et les idées. Dictionnaire des termes cadrant avec les idées, Bruxelles : Labor.

LaHaV R. (1989), “Against Compositionality: The Case of Adjectives”, Philosophical Studies: An International Journal for Philosophy in the Analytic Tradition 57 (3), 261-279.

LAMBRECHT K. (1984), "Frame Semantics, and Pragmatics in German Binomial Expressions", Language 60, 753-796.

LAMIROY B. et al. (2010), Les expressions verbales de la francophonie, Paris : Ophrys.

Le Fur D. (éd.) (2007), Dictionnaire des combinaisons de mots, Paris : Le Robert.

LEEMAN D. (1991), " Hurler de rage, rayonner de bonheur : remarques sur une construction en de ", Langue française 91, 80-101.

LÉON J. (2007), "Meaning by collocation. The Firthian filiation of Corpus Linguistics", in D. Kibbee (ed.), Proceedings of ICHoLS X, 10th International Conference on the History of Language Sciences, Amsterdam/Philadelphia: John Benjamins Publishing Company, 404-415.

MARTIN R. (1997), "Sur les facteurs du figement lexical ", in M. Martins-Baltar (éd.), La locution entre langue et usages, Fontenay-Saint-Cloud : ENS Éditions, 291-307.

MEJRI S. (1994), "Séquences figées et expressions de l'intensité. Essai de description sémantique ", Cahiers de lexicologie 65, 111-122.

Mel'Čuk I. (1998), “Collocations and Lexical Functions”, in A. Cowie (ed.), Phraseology. Theory, Analyses, and Applications, Oxford: Oxford University Press, 23-53.

MEL'ČUK I. (2003), "Collocations, définition, rôle et utilité ", in F. Grossmann \& A. Tutin (éds), Les collocations : analyse et traitement, Amsterdam : De Werelt, 23-32. 
MeL'ČUK I., CLAS A. \& Polguère A. (1995), Introduction à la lexicologie explicative et combinatoire, Louvain : Duculot.

MILNER G. (1969), " De l'armature des locutions proverbiales. Essai de taxonomie sémantique ", L'Homme 9 (3), 49-70.

MILNER J.-C. (1978), De la syntaxe à l'interprétation, Paris : Le Seuil.

Moon R. (1998), Fixed Expressions and Idioms in English: a Corpus-based Approach, Oxford: Oxford University Press.

Nesselhauf N. (2005), Collocations in a learner corpus, Amsterdam/Philadelphia: John Benjamins.

NunBerg G., SAG I. \& WaSoW T. (1994), “Idioms”, Language 70 (3), 491-538.

Palmer H. E. (1933), Second Interim Report on English Collocations, Tokyo : Kaitakusha.

SiePMANN D. (2006), "Collocations et dictionnaires d'apprentissage onomasiologiques bilingues: questions aux théoriciens et pistes pour l'avenir ", Langue française 107, 99-118.

SINCLAIR J. (1991), Corpus, Concordance, and Collocation, Oxford: Oxford University Press.

SMADJA F. (1993), “Retrieving Collocations from Text: Xtract”, Computational Linguistics 19 (1), 143-177.

SPANG-HANSSEN E. (1963), Les prépositions incolores du français moderne, Copenhague : Gads.

Spohr D. (2005), A Description Logic Approach to Modeling Collocations, PhD, Institut für Maschinelle Sprachverarbeitung, Universität Stuttgart (Germany).

SVENSSON M. H. (2004), Critères de figement. L'identification des expressions figées en français contemporain, Umeå : Umeå Universitet.

TUTIN A. (2008), "For an extended definition of lexical collocations", in E. Bernal \& J. DeCesaris (eds), Proceedings of the XIIIth EURALEX International Congress, Barcelona: IULA, 14531460.

TUTIN A. (2010), "Showing phraseology in context: an onomasiological access to lexicogrammatical patterns in corpora of French scientific writings", in S. Granger \& M. Paquot (eds), eLexicography in the 21st century: new applications, new challenges, Louvain-la-Neuve: Presses Universitaires de Louvain, 303-312.

Tutin A. \& Grossmann F. (2002), "Collocations régulières et irrégulières : esquisse de typologie du phénomène collocatif ", Revue française de Linguistique appliquée 7 (1), 7-25.

VAGUER C. (2006), "L'identité de la préposition dans : de l'intériorité à la coïncidence ", Modèles linguistiques 53, Tome XXVII-1, Toulon : Éditions des dauphins, 111-130.

VIVÈs R. (1993), "La prédication nominale et l'analyse par verbes supports ", L'Information grammaticale 59, 8-15.

WANNER L. (ed.) (1996), Lexical Functions in Lexicography and Natural Language Processing, Amsterdam/Philadelphia: John Benjamins.

Wierzbicka A. (1996), Semantics: Primes and Universals, Oxford: Oxford University Press.

WiLLIAMS G. (2003), "Les collocations et l'école contextualiste ", in F. Grossmann \& A. Tutin (éds), Les collocations: analyse et traitement, Travaux et recherches en linguistique appliquée, Amsterdam : de Werelt, 33-44.

WraY A. (2002), Formulaic Language and the Lexicon, Cambridge: Cambridge University Press. 